\title{
Percepções de crianças e adolescentes com e sem doença crônica de pele sobre saúde e envelhecimento
}

\section{Perceptions of children with and without chronic skin disease on health and aging}

\section{Percepción de los niños con y sin crônica de salud y envejecimiento dermatosis}

\author{
Fernanda Moehlecke* \\ Centro Universitário La Salle, Canoas, Rio Grande do Sul, Brasil
}

\section{Prisla Ücker Calvetti**}

Hospital de Clínicas de Porto Alegre - HCPA, Porto Alegre, Rio Grande do Sul, Brasil

\section{Magda Blessmann Weber***}

Universidade Federal de Ciências da Saúde de Porto Alegre - UFCSPA, Rio Grande do Sul, Brasil

\begin{abstract}
RESUMO
O universo infantil nos últimos anos vem ganhando visibilidade em diferentes campos. O crescente interesse de pesquisadores em conhecer as percepções das crianças acerca de diversos temas as tem destacado em suas pesquisas. O objetivo deste estudo foi investigar as percepções de crianças, sem e com doença crônica de pele, sobre saúde e envelhecimento. Trata-se de estudo qualitativo. Os participantes foram sete crianças sem doença crônica de pele e sete crianças com doença crônica de pele, onde o primeiro grupo possui vínculo com uma entidade comunitária do município de Gravataí e o outro com um ambulatório de saúde pública na cidade de Porto Alegre. Para a coleta de dados foi utilizado roteiro de entrevista semiestruturada. Para análise de conteúdo, foi utilizada a Análise de Bardin e a perspectiva do modelo biopsicossocial. As narrativas das crianças foram classificadas em três categorias a partir da avaliação de três juízes: Envelhecimento e Processo Saúde-doença, Prevenção e Envelhecimento, Relação entre Gerações. Destaca-se que as percepções dos grupos investigados apresentam semelhanças em relação ao envelhecimento, que representa a passagem do tempo. Sobre saúde, os grupos apresentam diferenças a partir de suas próprias experiências. Faz-se necessário o investimento em intervenções que promovam ações intergeracionais para promover a convivência saudável e a solidariedade entre as diferentes gerações.
\end{abstract}

Palavras-chave: crianças, envelhecimento, saúde, relações intergeracionais. 


\section{ABSTRACT}

The children's universe in recent years has been gaining visibility in different fields. The growing interest of researchers in understanding children's perceptions of various topics has highlighted them in their research. The objective of this study was to investigate the perceptions of children with and with chronic skin disease on health and aging. This is a qualitative study. The participants were 07 children without chronic skin disease and 07 children with chronic skin disease, where the first group had a link with a community entity in the city of Gravataí and the other with a public health clinic in the city of Porto Alegre. For data collection a semi-structured interview script was used. For content analysis, we used the Bardin Analysis and the perspective of the biopsychosocial model. The children's narratives were classified into three categories based on the evaluation of three judges: Aging and Health-Disease Process, Prevention and Aging, Relationship between Generations. It should be emphasized that the perceptions of the investigated groups present similarities in relation to aging, which represents the passage of time. On health, groups present differences from their own experiences. It is necessary to invest in interventions that promote intergenerational actions to promote healthy coexistence and solidarity between different generations.

Keywords: child, aging, health, intergenerational relationships.

\section{RESUMEN}

El universo infantil en los últimos años ha ido ganando visibilidad en diferentes campos. El creciente interés de los investigadores para conocer las percepciones de los niños sobre los diferentes temas que han destacado en sus investigaciones. El objetivo de este estudio fue investigar las percepciones de los niños y la enfermedad crónica de la piel en la salud y el envejecimiento. Se trata de un estudio cualitativo. Los participantes fueron 07 niños sin enfermedad crónica de la piel y 07 niños con enfermedad crónica de la piel, donde el primer grupo tiene vínculos con el municipio de Gravataí una entidad comunitaria y el otro con una clínica de salud pública en la ciudad de Porto Alegre. Para la recolección de datos se utilizó entrevistas semiestructuradas. Para el análisis de contenido se utilizó para el análisis de Bardin y la perspectiva del modelo biopsicosocial. Los relatos de los niños se clasificaron en tres categorías en función de la evaluación de tres jueces: Envejecimiento y Proceso Salud-Enfermedad, la prevención y el envejecimiento, las relaciones intergeneracionales. Es de destacar que las percepciones de los grupos investigados tienen similitudes en relación con el envejecimiento, que representa el paso del tiempo. La salud, los grupos difieren de sus propias experiencias. Es necesaria inversión en las intervenciones para promover actividades intergeneracionales para promover la sana convivencia y la solidaridad entre distintas generaciones.

Palabras clave: niños, envejecimiento, salud, las relaciones intergeneracionales. 


\section{I ntrodução}

O universo infantil, nos últimos anos, vem ganhando visibilidade em diferentes campos (Ramos, 2009). A criança, contudo, é considerada como um fenômeno social somente a partir dos anos 90, sendo entendida como uma categoria social autônoma, e analisável nas suas relações (Sarmento, 2005).

0 interesse crescente de pesquisadores de diferentes áreas em conhecer os pontos de vista das crianças acerca de diversos temas tem levado a colocá-las no centro das pesquisas por eles desenvolvidas, evidenciando a diversidade de sujeitos. As perspectivas infantis trazem à luz não apenas as particularidades da idade, mas, também, as experiências vividas em contextos específicos, as características de inserção social de seu grupo familiar, questões de gênero, etnia e cultura (Cruz, 2013).

As culturas infantis devem ser conhecidas a partir do que as próprias crianças dizem, pensam, sentem e fazem (Felipe, 1999). Talvez pouco se sabe sobre elas, porque pouco se ouve e pouco se pergunta às crianças (Quinteiro, 2002).

A partir destes princípios, este estudo apresenta a percepção de crianças sobre saúde e envelhecimento, considerando que, no campo da interação da criança com idoso, os estudos a este respeito são ainda escassos na literatura (Brandão, Smith, Sperb, \& Parente, 2006).

Importante salientar que, no Brasil, o ritmo de crescimento da população idosa tem sido sistemático e consistente. Segundo a última Pesquisa por Amostra de Domicílios - PNAD 2009, o país contava com uma população de 21 milhões de pessoas com 60 anos ou mais de idade (IBGE, 2010). Entretanto, o número de anos de vida saudável que podem ser esperados é de pelo menos 70 anos em apenas 25 países do mundo (Luchesi, Pavarini, \& Viana, 2012).

Essas estatísticas revelam alguns dos desafios na busca pelo bemestar global. Straub (2014) afirma que profissionais da saúde estão trabalhando para reduzir a discrepância de 30 anos em expectativa de vida entre países desenvolvidos e aqueles em desenvolvimento. Uma das ideias é de ajudar os adolescentes a fazerem uma transição segura e saudável para a idade adulta e alcançar uma compreensão mais profunda das relações entre gerações, gênero, etnicidade e saúde.

Para Luchesi et al. (2012), devido ao crescimento da convivência com idosos, destaca-se a importância da educação gerontológica, pois há a necessidade de um trabalho com as crianças sobre 0 envelhecimento, lembrando que um dia elas estarão cuidando de seus pais ou de seus avós. Também, é importante esse trabalho por considerar ainda que um dia elas se tornarão idosas, e seu 
comportamento nessa fase da vida pode ser influenciado pelas vivências e atitudes da infância.

A justificativa deste estudo, portanto, baseia-se pelo atual modo de vida das crianças, que, impactadas pelas alterações na estrutura familiar, convivem com o inevitável distanciamento dessas relações. Se as alterações familiares e a ausência de contato entre gerações são uma realidade para muitas crianças que não possuem algum tipo de doença crônica, o mesmo não é diferente para crianças que necessitam de tratamento médico sistemático. No caso de crianças com dermatite atópica (DA), por exemplo, vários aspectos da vida do paciente podem ser afetados pela doença, tais como a roupa a ser usada, a duração do banho, o uso contínuo de emolientes, vida familiar e social, a capacidade para a prática de esportes, sono ou estudar e, dependendo da idade, sexo e vida profissional. É uma doença inflamatória crônica da pele caracterizada por lesões intensamente pruriginosas e eczematosas. A localização geralmente depende da idade do paciente, sendo predominante na infância, mas pode ocorrer em qualquer idade (Amaral, March, \& Sant'Anna, 2012). A sobrecarga de cuidar de pacientes com DA pode levar a conflitos entre pais e irmãos saudáveis, o que pode alterar a estrutura familiar. O tratamento da DA é incomum, não só devido à dificuldade em relação aos aspectos clínicos, mas também por causa do investimento financeiro que os pais têm de fazer para manter cuidados de longa duração, o que muitas vezes torna difícil para as famílias para continuar com o tratamento (Amaral et al., 2012).

A maneira como o indivíduo constrói e interpreta as situações nas relações sociais produzem um efeito na sua saúde e bem-estar (França, Silva, \& Barreto, 2010). As pessoas que vivenciam aspectos positivos nas relações de apoio intergeracional sentem-se mais positivas em relação a si próprias e ao seu mundo, superando dificuldades inerentes à vida. As atividades de lazer, expressas nas mais variadas formas de cultura, guardam considerável potencial para aproximar diversas gerações como crianças, adolescentes, jovens adultos, adultos de meia idade e idosos; logo, estas atividades são efetivas estratégias de aproximação (Ferrigno, 2003). De acordo com Brandão et al. (2006), estudos a respeito da interação da criança com o idoso são ainda escassos na literatura, porém, se fazem necessários para compreender a percepção que um grupo tem sobre o outro, principalmente a percepção das crianças sobre os idosos.

Pensar em envelhecimento é um fato que geralmente igualamos a perdas. Perdas físicas, sociais, emocionais. Em todo o processo do desenvolvimento humano, apresentamos crises e desafios inerentes à vida, contudo, será que somos conscientes da passagem do tempo ou racionalizamos sobre o verdadeiro processo de envelhecimento? 0 que as crianças da segunda infância têm a dizer a respeito? Como crianças percebem este processo e como se relacionam com idosos? 
As percepções das crianças em dois contextos distintos refletem semelhanças ou diferenças?

Partindo destes questionamentos, esta pesquisa, através de um estudo qualitativo, objetiva investigar a percepção de dois grupos de crianças na fase da segunda infância, com idade entre seis e 10 anos, sobre saúde e envelhecimento. O primeiro grupo corresponde a crianças sem doença crônica de pele, participantes de um projeto recreativo em contexto comunitário, e o segundo, corresponde a crianças com doença crônica de pele, pacientes de um serviço de saúde pública ambulatorial.

\section{Método}

Trata-se de um estudo de caráter qualitativo descritivo exploratório, tendo como público:

a) Grupo 1: Sete crianças sem doença crônica de pele, participantes de atividades recreativas em contexto comunitário, com idade entre seis e 10 anos.

b) Grupo 2: Sete crianças com doença crônica de pele, participantes de atividades educativas e recreativas em um serviço de saúde pública ambulatorial, com idade entre seis e 10 anos.

Os dados foram coletados através de entrevista semiestruturada e de forma individual, em que cada criança foi convidada a "brincar de entrevista", um meio mais lúdico de envolvê-las no estudo. Método similar foi realizado para entrevistar crianças e adolescentes institucionalizados (Marzol, Bonafé, \& Yunes, 2012). Para tanto, respeitou-se, em primeira instância, a distinta rotina e sistemática de ambas as entidades, aonde, como critério de escolha, as primeiras sete crianças que chegaram aos respectivos locais no dia da coleta foram convidadas a participar do estudo.

As questões que nortearam a entrevista foram seguidas da seguinte forma, sendo complementadas de acordo com as narrativas e o momento de cada criança: O que é envelhecimento? / Para você, o que é saúde? / Para você, o que é doença? / Você já se imaginou uma pessoa idosa? / Você acha que pessoas idosas são doentes? / Você gosta de estar com pessoas idosas? / Você acha importante conviver com idosos? Por que?

As entrevistas foram gravadas e transcritas conforme análise de conteúdo de Bardin (1979). A análise de conteúdo é uma técnica que analisa o que foi dito nas entrevistas ou observado pelo pesquisador. $\mathrm{Na}$ análise do material, busca-se classificá-los em temas ou categorias que auxiliam na compreensão do que está por trás dos 
discursos (Cruz, 2008). O processo de explicitação, sistematização e expressão do conteúdo de mensagens, promovido pela análise de conteúdo, é organizado em três etapas, sendo estas realizadas em conformidade com três pólos cronológicos diferentes. De acordo com Bardin (1979), essas etapas compreendem: Pré-análise: consiste na escolha dos documentos a serem analisados, na retomada das hipóteses e dos objetivos iniciais da pesquisa. Exploração do material: consiste na operação de codificação, visando a transformação dos dados na compreensão do texto. Tratamento dos resultados obtidos e interpretação: consiste na interpretação dos resultados brutos, onde se propõem inferências e realizam-se interpretações previstas no quadro teórico pesquisado. Este estudo contou com a análise de conteúdo a partir de três juízes que analisaram o material de forma independente.

Respeitando os preceitos éticos da pesquisa com seres humanos, todos os participantes concordaram em colaborar com a pesquisa, assinaram o Termo de Consentimento Livre e Esclarecido (TCLE), bem como seus responsáveis, que assinaram o Termo de Consentimento Livre e Esclarecido. A pesquisa obteve aprovação pelo Comitê de Ética em Pesquisa do Centro Universitário La Salle, sob o parecer n. 804.444. As falas das crianças citadas nos resultados estão identificadas através de nomes fictícios a fim de preservar suas identidades.

\section{Resultados e Discussão}

A Tabela 1 apresenta as características das crianças da segunda infância participantes do estudo. 
Tabela 1.

Caracterização dos participantes.

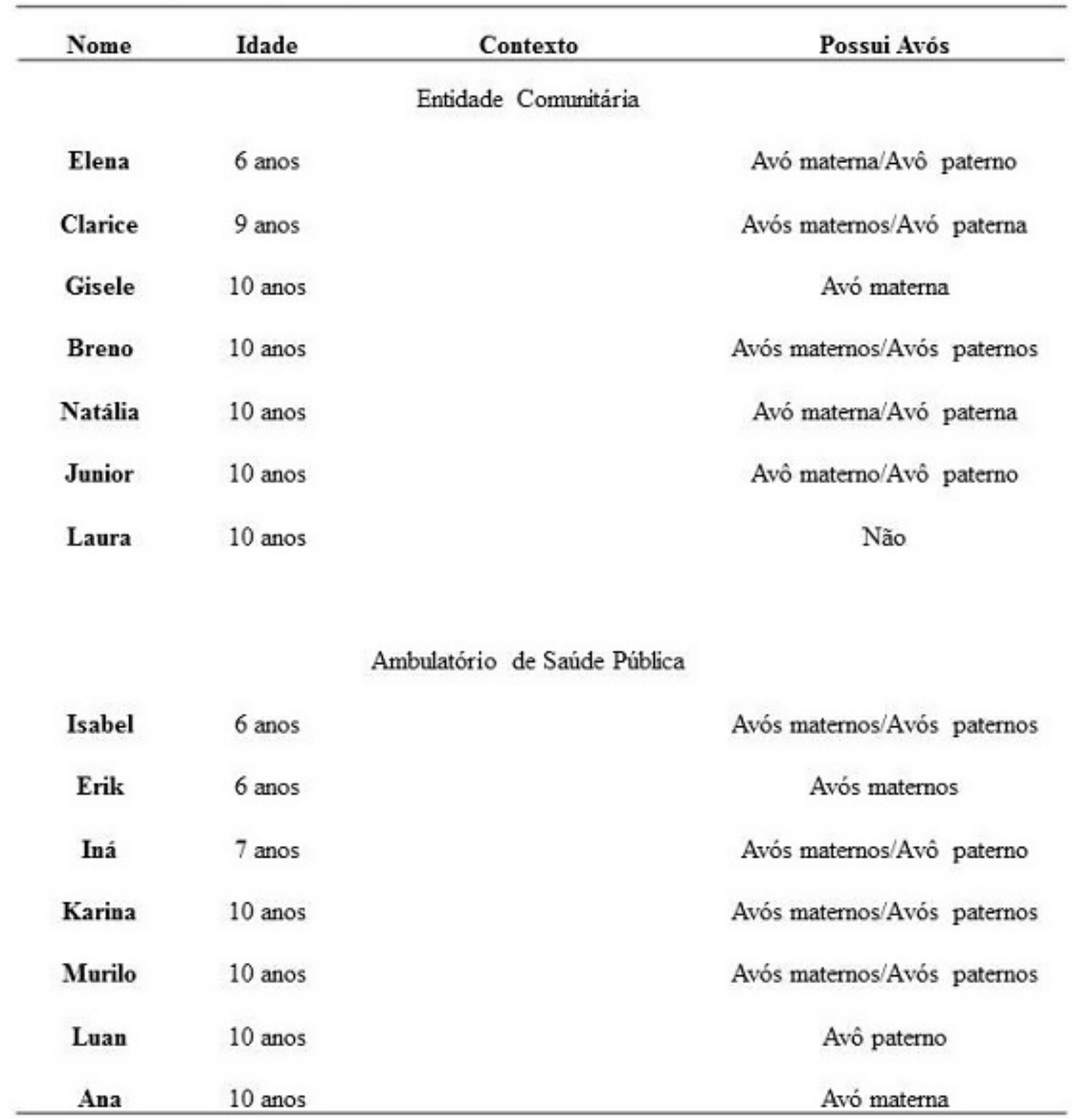

Envolver as crianças no processo deste estudo não foi tarefa complexa, pois, de pronto, ambos os grupos demonstraram interesse e curiosidade pela proposta, bem como apresentaram opiniões estruturadas e relevantes no que concerne a esta pesquisa. Assim como mencionam Zhang, Bécares, Chandola e Callery (2015), crianças a partir dos seis anos de idade podem demonstrar uma compreensão básica dos efeitos de investigação e o que se espera deles durante o processo de pesquisa. Ter um espaço para expressar suas opiniões vem ao encontro do que preconiza a sociologia da infância, que defende a criança como ator social e não apenas como a idade do não-falante, detentora de um discurso inarticulado, desarranjado ou ilegítimo (Sarmento, 2005). No estudo de Fernandes (2014), a autora menciona que a criança vem conquistando seu 
espaço no decorrer de cada geração e salienta o trabalho precursor na concepção de infância de Ariés, em que sinaliza que, entre os séculos XIX e XX, a criança passa a ganhar importância dentro do contexto familiar.

Assim sendo, a partir do conteúdo que emergiu durante as entrevistas, o mesmo foi analisado e distribuído em três categorias: 1) Processo Saúde-doença e Envelhecimento, 2) Prevenção e Envelhecimento, 3) Relação entre Gerações. Realizou-se por fim, o tratamento dos resultados apresentados, trazendo à luz as principais narrativas que respondessem aos objetivos do estudo.

\section{Processo Saúde-Doença e Envelhecimento}

De acordo com Straub (2014), a perspectiva biopsicossocial representa o ponto de vista ao qual a saúde e outros comportamentos são determinados pela interação entre mecanismos biológicos, processos psicológicos e influências sociais (mente-corpo). Sobre esta perspectiva, as narrativas das crianças destacaram as seguintes percepções sobre o envelhecimento:

"Quer dizer passar o tempo, passar os anos." (Elena, 6 anos/entidade comunitária).

"Para mim envelhecimento é quando a gente vai envelhecer aos poucos e a gente tem mais experiência da vida" (Breno, 10 anos/entidade comunitária).

“Envelhecer é quando a gente fica idoso ou quando a gente está na fase um pouco maior, a gente trabalha e tal ... a gente estuda no segundo grau, terceiro..." (Murilo, 10 anos/ ambulatório de saúde pública).

Tais percepções apresentam o discernimento de que o desenvolvimento humano é formado por ciclos. Conforme a lógica do desenvolvimento cognitivo de Piaget, é na terceira infância que há um aprimoramento das faculdades no campo qualitativo, onde o processo perceptivo desenvolve as capacidades de estruturação e reversibilidade mental e a dimensão temporal passa a ser mais perceptiva, tendo a criança à noção de sucessão temporal. Neste caso, as narrativas das crianças não apresentaram diferenças, pois ambos os grupos demonstraram entender que o processo de envelhecimento denota a passagem do tempo.

De acordo com Schneider e Irigaray (2008), a pessoa mais velha, geralmente, é definida como idosa quando chega aos 60 anos, independentemente de seu estado biológico, psicológico e social. 
Entretanto, para os autores, o conceito de idade é multidimensional e não é uma boa medida do desenvolvimento humano. A idade e o processo de envelhecimento possuem outras dimensões e significados que ultrapassam as dimensões da idade cronológica. É como nos apresenta uma das crianças:

"Envelhecer é aprender mais sobre a vida." (Gisele, 10 anos/entidade comunitária).

A fala da menina apresenta a percepção de que envelhecer está associado à sabedoria. $\mathrm{Na}$ visão de Platão (427-347 a.C.), a sabedoria possui três sentidos: sabedoria concebida (sophia); sabedoria prática (phronesis) e sabedoria com compreensão das coisas (episteme). Os três demandam tempo e estão intimamente associadas à idade (Vieira, 2010).

Sobre velhice, ao serem incentivados a descreverem uma pessoa idosa, destacaram, em sua maioria, a característica da pele de uma pessoa idosa:

“A pele fica molinha." (Elena, 6 anos/entidade comunitária).

"A pele é mais fina." (Gisele, 10 anos/entidade comunitária).

"A pele é murcha." (Erik, 6 anos/ambulatório de saúde pública).

A pele é considerada um dos órgãos que mais sofre transformações à medida que a idade avança. O envelhecimento pode ser definido como um processo biológico no qual ocorrem alterações das características morfológicas e fisiológicas no organismo vivo ao longo do tempo. A pele apresenta, com o avançar da idade, diminuição da espessura epiderme-derme; redução da elasticidade e da secreção de sebo pelas glândulas sebáceas; resposta imunológica comprometida; decréscimo do número de glândulas sudoríparas; diminuição do leito vascular com fragilidade dos vasos sanguíneos (Oriá, Brito, Ferreira, Santana, \& Fernandes, 2003). Clinicamente, o envelhecimento intrínseco se expressa como uma pele enrugada, flácida e seca (Brandão et al., 2006). Assim menciona uma das crianças:

"A pele é ressecada." (Murilo, 10 anos/ambulatório de saúde pública).

Nessa última narrativa, ressalta-se que, ao descrever a pele de um idoso como ressecada, Murilo traz uma característica também da sua pele, pois é portador de um tipo de doença crônica que ocasiona o ressecamento cutâneo. 
Outras características se apresentaram:

"Tem cabelo cinza e não tem dente." (Iná, 7 anos/ambulatório de saúde pública).

"Aparência de quem não é novo." (Clarice, 9 anos/entidade comunitária).

A partir destas narrativas, é possível destacar as manifestações somáticas da velhice, última fase do ciclo de vida caracterizada pela redução da capacidade funcional, calvície, canície, redução da capacidade de resistência entre outras (Netto, 2007). Sendo assim, constatou-se que, as crianças demonstraram entendimento sobre a diferença do processo de envelhecimento e do estágio da velhice. Outro fator relevante foi o fato de que não houve diferenças sobre esta percepção entre as crianças sem doença crônica de pele e das crianças com doença crônica de pele.

Outra percepção que emergiu foi o aspecto emocional na relação com pessoas mais velhas. Assim como defende Vygotsky (como citado em Wortmeyer, Silva, \& Branco, 2014), o afeto se mantém essencial ao longo de todo o desenvolvimento da criança. Os impulsos afetivos são os acompanhantes permanentes de cada ciclo no desenvolvimento desta, ou seja, o afeto inicia e encerra o processo de desenvolvimento psíquico e a formação de sua personalidade. Este aspecto destacou-se através das falas de Clarice:

“Ah, aquelas pessoas que são legais, bem disponíveis eu adoro, mas se são aquelas que não dão atenção, sabe? Eu não gosto, depende... Aqueles que sentem o que a criança diz, dá atenção sim...é que eu não gosto muito da minha avó, eu prefiro os que são legais." (Clarice, 9 anos/entidade comunitária).

$\mathrm{Na}$ tentativa de compreender como as emoções modificam o comportamento, Vygotsky (como citado em Wortmeyer et al., 2014) não deixa de tomar como premissa a origem biológica das emoções: "... tudo nos permite afirmar que é verdade que a emoção é um sistema de reações vinculado de modo reflexo aos estímulos" (Burlá, Pessini, Siqueira, \& Nunes, 2014, p. 115). Nesse ponto, o autor identifica que a reação emocional é "um poderoso organizador do comportamento" (Burlá et al., 2014, p. 118), onde a afetividade tem um papel crucial no processo de aprendizagem do ser humano, pois está presente em todas as áreas da vida, influenciando profundamente o crescimento cognitivo (Wortmeyer et al., 2014). Crianças de ambos os grupos relataram gostar da companhia de idosos. 
No que se refere às percepções de saúde e doença, Straub (2014) afirma que, há muito tempo, psicólogos reconhecem que cada idade tem seu modo especial de enxergar o mundo, reforçando que os conceitos de doença na infância, muitas vezes, incluem noções mágicas sobre a causalidade. Somente quando seu conceito de auto eficácia continua a amadurecer, elas começam a compreender que podem tomar medidas para controlar sua saúde. Portanto, foi possível observar que tanto as crianças sem doença crônica de pele quanto as crianças com doença crônica de pele demonstraram entendimento sobre saúde e doença. Talvez, o que tenha se apresentado mais evidente foi o fato de que crianças sem doença de pele citaram exemplos de pessoas próximas que estão ou estiveram doentes, ao passo que as crianças com doença de pele foram mais explícitas em suas colocações devido ao fato provável de conviverem com uma rotina médica sistemática, conforme as seguintes colocações:

"Doença é quando a pessoa descobre que está ruim, que tem que se preocupar mais com ela." (Clarice, 9 anos/entidade comunitária).

"Doença é dermatite atópica, câncer, bastante doenças." (I ná, 7 anos/ambulatório de saúde pública).

Além disso, sobre saúde e doença, foi possível observar que para as crianças sem doença crônica de pele, saúde está relacionada a bons hábitos. Já o grupo com doença crônica de pele atribuiu saúde à beleza física e até mesmo a um posto de saúde, retratando mais uma vez suas experiências com a doença e rotinas médicas. De acordo com as crianças:

"Saúde é quando tu sabes que está comendo bem, correndo, fazendo exercício, curtindo a vida." (Clarice, 9 anos/entidade comunitária)

"Saúde é se cuidar, não comer muita besteira, comer mais frutas." (Gisele, 10 anos/entidade comunitária)

"Pessoa que é saudável é legal e é bonita." (Isabel, 6 anos/ambulatório de saúde pública).

"Saúde é um posto de saúde... pessoa com saúde é magra." (Iná, 7 anos/ambulatório de saúde pública).

De qualquer forma, todas as crianças afirmaram que nem todos os idosos são doentes e consideram que doenças fazem parte da vida, e 
algumas são mais frequentes na velhice. Portanto, nesta primeira categoria, foi possível perceber que não há diferenças nas narrativas das crianças sem doença crônica de pele e das crianças com doença crônica de pele sobre saúde, doença e envelhecimento. Além disso, as crianças demonstraram possuir uma concepção bem clara dos problemas que geram a situação de doença; provavelmente, a experiência com familiares doentes, ou elas próprias, tenha marcado essa percepção.

\section{Prevenção e Envelhecimento}

De acordo com Straub (2014), geralmente pensamos em prevenção para modificar o risco da pessoa antes que a doença a atinja. Este autor ainda cita que muitos pesquisadores diferenciam três tipos de prevenção, que são realizados antes, durante e depois de uma doença atacar, sendo elas: prevenção primária, prevenção secundária e prevenção terciária.

No caso das crianças deste estudo, a prevenção fora mencionada, em sua maioria, de forma primária e secundária, ao qual as ações que promovem saúde são realizadas para prevenir a instalação de determinada doença ou identificada e tratada no começo do seu curso. Exemplificam a boa nutrição e a prática de exercícios como fatores primordiais para uma boa saúde e um consequente envelhecimento saudável, como mencionaram:

"Se cuidar, não comer muita besteira, comer mais frutas." (Gisele, 10 anos/entidade comunitária).

"Comer bem, tomar os remédios se ficar um pouco doente." (Murilo, 10 anos/ambulatório de saúde pública).

Conforme Straub (2014), comportamentos de saúde são comportamentos das pessoas para melhorar ou manter sua saúde. Prática de exercícios, uso de protetor solar, dieta com baixo teor de gordura, dormir bem, uso de cinto de segurança são comportamentos que auxiliam a imunizar as pessoas contra doenças e ferimentos. A prática de exercícios físicos e a alimentação saudável também se fez presente na fala de Clarice como um comportamento preventivo:

“Alimentação saudável, exercícios, visitar o médico e cuidar da vida." (Clarice, 9 anos/entidade comunitária).

A manutenção de um estado nutricional adequado é essencial para uma boa saúde. O envelhecimento adequado está associado a mudanças na composição corporal em decorrência de mudanças na 
fisiologia, no metabolismo e na demanda nutricional (Santos, Freitas, Cançado, \& Gorzoni, 2014). Além disso, para Straub (2014), a prática de exercícios é o mais próximo que podemos chegar da fonte da juventude, tornando-se mais importante à medida que as pessoas envelhecem, devido ao fato de promover bem-estar físico, psicológico, além de desacelerar os efeitos do envelhecimento. A prática regular de exercícios pode reduzir o risco de doenças cardiovasculares, diabetes e outras condições relacionadas ao estresse. Além disso, alguns estudos apontaram que fazer exercícios protege contra a osteoporose, doença caracterizada pelo declínio na densidade óssea devido à perda de cálcio. O autor ainda destaca que hábitos de saúde costumam ser herdados dos pais e pessoas próximas que refletem modelos para os comportamentos de saúde. Mesmo havendo uma base genética, as crianças podem adquirir expectativas sobre um mau comportamento de saúde observando seus familiares.

Os hábitos alimentares desenvolvidos durante a infância podem persistir na adolescência e idade adulta, influenciando o crescimento individual, no desenvolvimento e na saúde (Zhang et al., 2015). Para as crianças, as suas decisões alimentares são muitas vezes feitas dentro do contexto familiar, que é o aspecto mais influente do contexto social imediato.

Assim como na primeira categoria, foi possível observar que não há diferenças nas percepções de crianças sem doença crônica de pele e de crianças com doença crônica de pele no que se refere aos cuidados e hábitos saudáveis para a manutenção de uma vida um consequente envelhecimento adequado.

\section{Relação entre Gerações}

Erikson, no seu modelo de desenvolvimento psicossocial ao longo da vida, foi o primeiro autor a introduzir o termo "generatividade", no sétimo estágio de vida, como o contraponto à estagnação na meia idade. Em seu modelo, o autor destaca a importância do relacionamento intergeracional harmonioso ao longo da vida. A generatividade é definida por ele como uma tarefa social importante para a vida adulta, necessária para a transição bem-sucedida à fase final de integridade (França et al., 2010). Cícero, em De senectude, quando considerou a velhice a presença do passado no presente, qualifica os idosos como colaboradores competentes para tornar harmônica a vida em sociedade (Burlá et al., 2014). Esta consideração, também ficou evidente nas seguintes percepções: 
"Eles nos ensinam mais, eles falam para nós o que já aprenderam e o que a gente pode aprender." (Gisele, 10 anos/entidade comunitária).

"Quando tem um adolescente, acho que ele se sente mais fortalecido, porque ele não será aquela pessoa que ninguém gosta." (Gisele, 10 anos/entidade comunitária).

“Para a saúde, eles ficam mais felizes né, ao invés de ficarem lá se deprimindo em casa, no sofá... acho bem melhor eles estarem com as crianças para ver como foi a vida deles." (Clarice, 9 anos/entidade comunitária).

Especialmente nessas narrativas, as crianças trazem dois aspectos relevantes: o idoso se sente mais fortalecido com a presença dos jovens, e a convivência entre estas gerações pode trazer consequências benéficas à saúde deste. Durante a velhice, as pessoas possuem maior probabilidade de serem socialmente isoladas, devido a problemas de saúde, baixa moral e dificuldades de comunicação. Manter idosos saudáveis e ativos como membros vitais de suas comunidades é um desafio emergente da sociedade em envelhecimento (Morita \& Kobayashi, 2013). O valor do contato das crianças com os idosos é irrefutável para o resgate de valores, para a noção de tempo, da transformação ao longo dos anos e da identidade familiar (França et al., 2010). Todas as atividades que envolvam idosos e crianças devem ser permeadas pelo diálogo, em que precisam estar presentes as situações reais da comunidade, 0 processo de envelhecimento e as medidas a serem tomadas pela sociedade para garantir a mobilidade, a participação social e a independência daquele que envelhece (OPAS, 2005). Este tipo de atitude também foi observado pelas crianças:

"Gosto daqueles que são disponíveis, nem todos são legais." (Clarice, 9 anos/entidade comunitária).

“O jovem pode aprender com o idoso porque o idoso já passou por todos por todas as fases e ele já sabe tudo e o jovem ainda tá aprendendo." (J unior, 10 anos/entidade comunitária).

O contato e a comunicação entre crianças e idosos permite o apoio mútuo através da escuta, reflexão e a troca de conselhos, incluindo a manutenção de um senso de identidade, o alívio contra a solidão, a depressão e a ansiedade (Morita \& Kobayashi, 2013). Se a imagem da velhice for sustentada com base na noção de declínio, isto também poderá gerar consequências negativas não apenas para os idosos frente ao envelhecimento, mas, também, para aquelas 
pessoas que ainda não são idosas (Brandão et al., 2006). De acordo com dados do Instituto Brasileiro de Geografia e Estatística (IBGE, 2010), existe, atualmente no Brasil, mais de 21 milhões de idosos, e embora o envelhecimento populacional seja uma conquista da humanidade, é um fenômeno que tem consequências socioculturais e político-econômicas, sendo um grande desafio elaborar e implantar políticas públicas que promovam com qualidade e equidade à longevidade da população (Netto, 2007). Programas intergeracionais podem ser uma possibilidade para essa interação saudável. Para os idosos, os efeitos destes programas incluem o aumento da autoestima, melhoria do bem-estar, o aumento do contato social e a diminuição da angústia, enquanto atitudes positivas para com os idosos e compreensão do processo de envelhecimento podem ser benefícios encontrados pelas crianças (Burlá et al., 2014).

Ainda sobre a relação de jovens e adultos destaca-se a seguinte narrativa, que apresenta uma opinião divergente:

"Porque são pessoas boas, experientes que, já passaram por muitas coisas, o jovem não faz nada de especial para o idoso." (Murilo, 10 anos/ambulatório de saúde pública).

A maneira como a sociedade se comunica com o idoso produz forte impacto no uso da linguagem por este (Marzol et al., 2012). Assim sendo, acredita-se que, ao compartilhar as experiências entre idosos e jovens, está se combatendo o preconceito etário, o que pode contribuir para uma sociedade mais justa, tolerante e solidária.

Nesta categoria, ficou evidente, em ambos os grupos, a importância das relações positivas com as demais fases do desenvolvimento humano, especialmente com idosos. As possibilidades de convívio entre estas distintas gerações são infinitas, de acordo com as percepções das crianças aqui apresentadas e, segundo elas, é uma forma natural de perpetuar a cultura e experiências vividas.

\section{Considerações finais}

Compreender desde cedo de que forma o ser humano desenvolve-se, seus distintos processos e mecanismos é um fator que pode ser primordial para um desenvolvimento humano adequado, além de contribuir significativamente para a prevenção de possíveis problemas inerentes à vida, como as doenças e suas consequências físicas, emocionais e mentais. Investigar como crianças percebem estes processos pode refletir discursos e comportamentos repassados por distintas gerações e, a partir disso, favorecer meios de educação para a saúde em diferentes contextos. Através das percepções das crianças deste estudo, foi possível perceber que o processo de 
envelhecimento deve ser tratado em sua integralidade, pois o homem é compreendido como um ser em constante desenvolvimento dentro de distintos aspectos e deve deter-se no autocuidado, no cuidado com o outro e consequentemente no cuidado com a sociedade. Entender o envelhecimento como um processo, a velhice como um estágio que representa sabedoria, alegria e também declínio físico, e o processo saúde-doença como consequência de um estilo de vida, denota um conhecimento da realidade e do meio ao qual estas crianças estão inseridas e que nos faz pensar se a sabedoria realmente está associada somente aos idosos.

Ao iniciar um trabalho ainda na infância sobre o envelhecimento e o processo saúde-doença, o adulto tem a oportunidade de resinificar o desenvolvimento das mesmas e do seu próprio meio. Aprender a lidar com estes processos pode conscientizar as crianças de que suas ações possivelmente influenciarão o modo como vão envelhecer. As próprias crianças deste estudo demonstraram isso: apesar de não pensarem no seu desenvolvimento a médio e longo prazo, entendem a necessidade de cultivar hábitos saudáveis para uma boa qualidade de vida. Crianças pensam no agora, contudo, se possuírem um presente estruturado, com ações educativas embasadas que denotem leveza e apreciação à vida, serão pessoas que se responsabilizarão pelo ciclo vital de forma eficaz e favorável. Ao mesmo tempo em que se divulga um discurso que defende que a criança é o futuro e que devemos respeitar o idoso, nossa memória, o passado, a crise econômica nacional tende a ser deficitária exatamente na infância e na velhice e pouco se faz efetivamente no sentido de escutar estas duas gerações, já que o adulto domina o presente de forma mais objetiva e ágil. No contexto educacional, isso também não é diferente, pois também é priorizado o domínio de habilidades e conteúdo, negligenciando-se a necessidade de compreensão mais profunda das relações entre o indivíduo, os outros e o seu meio.

Esta é uma missão que adultos e idosos devem se responsabilizar, pois estimular a interação entre diferentes gerações tende a favorecer a troca de conhecimentos e a formação de relações mais positivas. Estudos nesta área são escassos e se fazem necessários considerando os aspectos acima citados. Diferentes áreas técnicas podem envolver-se nesta caminhada e assim contribuir para um desenvolvimento humano integral e mais saudável. 


\section{Referências}

Amaral, C. S. F. D., March, M. D. F. B. P., \& Sant'Anna, C. C. (2012). Quality of life in children and teenagers with atopic dermatitis. Anais Brasileiros de Dermatologia, 87(5), 717-723.

Bardin, L. (1979). Análise de conteúdo. Lisboa: Edições 70.

Brandão, L., Smith, V., Sperb, T. M., \& Parente, M. A. D. M. P. (2006). Narrativas Intergeracionais. Psicologia: Reflexão e Crítica, 19(1), 98-105.

Burlá, C., Pessini, L., Siqueira, J. E., \& Nunes, R. (2014). Envelhecimento e doença de Alzheimer: reflexões sobre autonomia e o desafio do cuidado. Revista Bioética, 22(1), 8593.

Cruz, S. H. V. (2008). A criança fala: A escuta de crianças em pesquisas. São Paulo: Editora Vozes.

Cruz, S. H. V. (2013). A criança fala: a escuta de crianças em pesquisas. Cadernos de Pesquisa, 39(136), 330-330.

Felipe, J. (1999). Entre as Tias e Tiazinhas: Pedagogias Culturais em Circulação. In L. H. Silva (Org.), Século XXI: Qual conhecimento? Qual currículo? (pp. 133-166). Rio de Janeiro: Vozes.

Fernandes, L. S. (2014). O Idoso e a Intergeracionalidade com o Público Infantil a partir do Projeto "Era Uma Vez... Atividades Intergeracionais" Realizado pelo SESC-CE através do TSI (Trabalho de Conclusão de Curso não publicado). Fortaleza, Faculdade Cearense.

Ferrigno, J. C. (2003). Co-Educação entre Gerações. São Paulo: Vozes.

França, L. H. D. F. P., Silva, A. M. T. B. D., \& Barreto, M. S. L. (2010). Programas intergeracionais: quão relevantes eles podem ser para a sociedade brasileira. Revista Brasileira de Geriatria e Gerontologia, 13(3), 519-531.

Instituto Brasileiro de Geografia e Estatística (2010). Censo 2010 [Internet]. Brasília: IBGE. Retrieved from http: //censo2010.ibge.gov.br

Luchesi, B. M., Pavarini, S. C. I., \& Viana, A. S. (2012). Alterações cognitivas de idosos no contexto domiciliar e atitudes de crianças em relação à velhice. Revista da Escola de Enfermagem da USP, 46(2), 335-341.

Marzol, R. M., Bonafé, L., \& Yunes, M. A. M. (2012). As perspectivas de crianças e adolescentes em situação de acolhimento sobre os cuidadores protetores. Psico (Porto Alegre), 43(3), 317-324.

Morita, K., \& Kobayashi, M. (2013). Interactive programs with preschool children bring smiles and conversation to older adults: time-sampling study. BMC geriatrics, 13(1), 02-08. 
Netto, M. P. (2007). Tratado de gerontologia (2nd. ed.). São Paulo: Atheneu.

Organização Pan-Americana De Saúde - OPAS. (2005). Envelhecimento ativo: Uma política de saúde. Brasília: OPAS.

Oriá, R. B., Brito, G. A., Ferreira, F. V. A., Santana, É. N., \& Fernandes, M. R. (2003). Estudo das alterações relacionadas com a idade na pele humana, utilizando métodos de histomorfometria e autofluorescência. Anais Brasileiros de Dermatologia, 78(4), 425-434.

Quinteiro, J. (2002). Infância e Educação no Brasil: Um Campo de Estudos em Construção. In A. G. Faria, Z. B. P. Dermatini, \& P. Dias (Org.), Por uma Cultura da Infância: Metodologia da Pesquisa com Crianças (pp. 19-47). São Paulo: Autores Associados.

Ramos, A. C. (2009). Cultura Infantil e Envelhecimento: O que Crianças têm a dizer sobre a Velhice? Um Estudo com meninos e meninas da Periferia de Porto Alegre (Dissertação de mestrado não publicada). Porto Alegre, Universidade Federal do Rio Grande do Sul.

Santos, V. H., Freitas, E. V., Py, L., Cançado, F. A., Gorzoni, M. L. (2014). Tratado de Geriatria e Gerontologia (3rd. ed). Rio de Janeiro: Guanabara Koogan.

Sarmento, M. J. (2005). Gerações e alteridade: interrogações a partir da sociologia da infância. Educação e Sociedade, 26(91), 361378.

Schneider, R. H., \& Irigaray, T. Q. (2008). O envelhecimento na atualidade: aspectos cronológicos, biológicos, psicológicos e sociais. Estudos em psicologia, 25(4), 585-593.

Straub, R. O. (2014). Psicologia da Saúde: Uma Abordagem Biopsicossocial (3rd. ed). Porto Alegre: Artmed.

Vieira, S. C. L. (2010). Paredes que Separam Gerações: Crianças e Idosos em Instituições (Dissertação de mestrado não publicada). Aveiro, Portugal, Departamento de Educação Universidade de Aveiro.

Wortmeyer, D. S., Silva, D. N. H., \& Branco, A. U. (2014). Explorando o território dos afetos a partir de Lev Semenovich Vigotski. Psicologia em Estudo, 19(2), 285-296.

Zhang, N., Bécares, L., Chandola, T., \& Callery, P. (2015). Intergenerational differences in beliefs about healthy eating among carers of left-behind children in rural China: A qualitative study. Appetite, 95, 484-491. 


\section{Endereço para correspondência}

Fernanda Moehlecke

Centro Universitário La Salle

Av. Vítor Barreto, 2288 - Centro, CEP 92010-000, Canoas - RS, Brasil

Endereço eletrônico: nanda.ief@hotmail.com

\section{Prisla Ücker Calvetti}

Hospital de Clínicas de Porto Alegre - HCPA

Rua Ramiro Barcelos, 2350, Santa Cecília, CEP 90035-903, Porto Alegre - RS, Brasil

Endereço eletrônico: prisla.calvetti@gmail.com

\section{Magda Blessmann Weber}

Universidade Federal de Ciências da Saúde de Porto Alegre - UFCSPA

R. Sarmento Leite, 245, Centro Histórico, CEP 90050-170, Porto Alegre - RS, Brasil

Endereço eletrônico: mbw@terra.com.br

Recebido em: 15/02/2016

Reformulado em: 10/03/2017

Aceito para publicação em: 24/03/2017

\section{Notas}

* Educadora Física. Mestre em Saúde e Desenvolvimento Humano - Mestrado Profissional Universidade La Salle Canoas/RS.

** Psicóloga. Doutora em Psicologia - PUCRS. Pós-doutorado em Medicina: Ciências Médicas - UFRGS.

*** Médica Dermatologista. Mestre em Ciências Médicas: Pediatria - UFRGS. Doutorado em Saúde da Criança e do Adolescente - UFRGS.

Este artigo de revista Estudos e Pesquisas em Psicologia é licenciado sob uma Licença Creative Commons Atribuição-Não Comercial 3.0 Não Adaptada. 